\title{
DIVERSIFICACIÓN DE ITINERARIOS DE EVALUACIÓN EN EDUCACIÓN SUPERIOR. UNA PROPUESTA COLABORATIVA DE INNOVACIÓN EDUCATIVA ${ }^{1}$
}

Diversification of evaluation itineraries in Higher Education. A collaborative proposal of educational innovation

Diversificação de avaliação itinerários no ensino superior. A proposta colaborativa de inovação educacional

\section{Daniel Martos Garcia}

Universitat de València, España. Telf.: +34963983383. Correo electrónico:

daniel.martos@uv.es

\section{Alexandra Valencia-Peris}

Universitat de València, España. Telf.: +34963828920. Correo electrónico: alexandra.valencia@uv.es

\section{Vicente Miñana Signes}

Universitat de València, España. Telf.: 961625095. Correo electrónico:

vicente.minana@uv.es

\section{Rodrigo Atienza Gago}

Universitat de València, España. Telf.: +34961625095. Correo electrónico: rodrigo.atienza@uv.es

\section{Enric Monforte i Casañ.}

Universitat de València, España. Telf.: 963864695. Correo electrónico: enric.monforte@uv.es

\footnotetext{
${ }^{1}$ Este estudio se ha llevado a cabo dentro del proyecto de I+D+i: "La evaluación en la formación inicial del profesorado de educación física". Convocatoria del Plan nacional de Proyectos I+D+i (2008-2011) (BOE del 31/12/2009). Proyectos de Investigación Fundamental no orientada. Referencia: EDU 2010-19637 (Subprograma EDUC) de 3 años de duración (2010-2012).
} 


\title{
Resumen
}

La sociedad en general y la educación en particular demandan nuevas formas de plantear el proceso de enseñanza y aprendizaje que deben estar en consonancia con los actuales paradigmas educativos y con las demandas sociales. Una estrategia de cambio a adoptar es la implementación de innovaciones educativas de forma colaborativa. En este caso, se describe un sistema de evaluación aplicados en Educación Superior en el cual se establecen tres itinerarios en función del grado de asistencia del alumnado: formativo, mixto y final. Esta experiencia ha supuesto una excelente oportunidad para tomar conciencia sobre la evaluación y para reflexionar de forma crítica y colaborativa sobre nuestra labor docente. Palabras clave: Educación Física, evaluación formativa, formación docente, innovación educativa.

\begin{abstract}
Society at large and education in particular demand new ways of thinking about the learning and instruction process, which must be in relation with the current educational paradigms and with the social claims. A change strategy to take into account is the implementation of educational innovations collaboratively. In this case, an evaluation system in Higher Education divided in three itineraries depending on the students' attendance is described: formative, mixed and final. This experience has supposed an excellent opportunity to be aware about evaluation and to reflect critically and collaboratively on our educational work. Keywords: Physical Education, formative assessment, teacher education, educational innovation.
\end{abstract}

\section{Resumo}

A sociedade em geral e da educação, em particular, a procura de novas maneiras de abordar o processo de ensino e aprendizagem que deve estar em consonância com paradigmas educacionais atuais e demandas sociais. A estratégia de mudança para adotar é implementar inovações educacionais de forma colaborativa. Neste caso, um sistema de avaliação na Educação Universitaria dividido em três itinerários dependendo do grau de atendimento dos alunos da escola: formação, misturado e final. Esta experiência tem sido uma excelente 
oportunidade de aumentar a consciência da avaliação e refletir criticamente e de forma colaborativa em nosso ensino.

Palavras chave: educação física, avaliação formativa, formação de professores, a inovação educacional.

\section{Introducción}

Más allá del debate sobre un modelo u otro de organización y estructura de la universidad, la construcción del Espacio Europeo de Educación Superior (EEES) se ha convertido en una excelente excusa para remover los aletargados cimientos universitarios (Benito \& Cruz, 2003). Sin embargo, para algunos de estos cambios se revela más apropiado poner encima de la mesa los nuevos paradigmas de la educación, algunos de ellos ${ }^{2}$ recogidos en los documentos que sustentan dicho EEES, y que no hacen sino focalizar la atención en los procesos de enseñanza y, sobre todo, de aprendizaje, de forma que "se enseñe a los alumnos a pensar, hablar y hacer" (Gijón \& Crisol, 2012: 403).

Una de las áreas de actuación que más dinamismo atesora en las últimas décadas es la evaluación, como lo atestigua el incremento de publicaciones realizadas sobre el tema (Arribas, 2012). Posiblemente, dados estos cambios de paradigma educativo, sea la evaluación uno de los aspectos que más necesitaba de nuevos enfoques para separarla de la mera calificación y, fundamentalmente, para darle una orientación más educativa, puesto que, como argumentan Azaustre y Monescillo (2013), la evaluación es un elemento curricular clave y, por ello, debe integrase plenamente en dicho proceso educativo dando lugar a un alineamiento curricular (Biggs, 2005). Afortunadamente, la evaluación para el aprendizaje ha experimentado en los últimos años un considerable empuje, tanto en sus fundamentos teóricos como en sus propuestas de aplicación práctica. Así, encontramos en la literatura una cantidad ingente de trabajos dedicados a la evaluación auténtica, la evaluación educativa, para el aprendizaje o la evaluación formativa. Es este último concepto es el que usamos en esta propuesta, bajo el paraguas de la Red de Evaluación Formativa y Compartida en Educación Superior, constituida durante el curso 2005-2006 y a la que pertenecemos. Dicha

\footnotetext{
${ }^{2}$ Destacan una serie de cambios respecto a: i) el papel que juega alumnado y profesorado en el proceso de enseñanza y aprendizaje; ii) cambios a nivel metodológico; y iii) cambios a nivel de evaluación. Para profundizar en estos temas puede consultarse Benito y Cruz (2003), Hamodi (2016) y López-Pastor (2009).
} 
red está formada en la actualidad por 98 profesores y profesoras de 28 universidades (mayoritariamente españolas aunque recientemente se han incorporado universidades de Chile, México y Ecuador) distintas y trata de coordinar al profesorado que desarrolla innovaciones en el campo de la evaluación compartida y formativa (López-Pastor et al. 2011). La evaluación formativa y compartida, en este sentido, se entiende como aquella cuya finalidad "es la mejora del proceso [de enseñanza-aprendizaje] y/o de las personas que están implicadas en el proceso" (López-Pastor, 2011: 25).

Los postulados de la evaluación formativa y compartida son atingentes a una visión de la educación preocupada por los procesos de aprendizaje y por la participación del alumnado en dichos procesos, en este caso, específicamente en la evaluación y, consecuentemente, en su aprendizaje. Así, para una educación significtiva que trascienda los límites del aula, se revela necesario que alumnos y alumnas aprendan a tomar decisiones sobre su aprendizaje, se responsabilicen y, en el caso de la formación del profesorado, interioricen dichos aprendizajes para, en un futuro, trasladarlos como consideren a su alumnado.

La evaluación formativa supone el reconocimiento de la inherente relación entre educación y evaluación; por el contrario, tenemos la evaluación sumativa, aquella que se preocupa por "hacer una revisión y un juicio de valor sobre cuánto se sabe, cuánto se ha mejorado..." (López-Pastor, 2011: 25). Asimismo, la evaluación final es aquella que se presenta como un derecho legal derivado del hecho de matricularse en una asignatura y que ofrece al alumnado la posibilidad de superar la materia acreditando unos conocimientos que no ha adquirido con el seguimiento y asistencia a las sesiones planteadas por el profesorado, sino con la superación, normalmente, de un examen final.

En las últimas décadas se han hallado numerosas evidencias de las ventajas ${ }^{3}$ que suponen los sistemas de evaluación formativa y compartida en los procesos de enseñanzaaprendizaje tanto para el alumnado como para el profesorado (Buscà, Pintor, Martínez y Peire, 2010; López-Pastror, Pintor, Muros y Webb, 2013; Manrique, Vallés y Gea, 2012; Vallés, Ureña y Ruiz, 2011; Zaragoza, Luis-Pascual y Manrique, 2009) (Tabla 1).

\footnotetext{
${ }^{3}$ Para profundizar en las ventajas y dificultades de los sistemas de evaluación formativa y compartida se recomienda la lectura de la profusa revisión recogida en Hamodi (2016: 33-39).
} 
Tabla 1. Ventajas de los sistemas de evaluación formativa y compartida

\begin{tabular}{|c|c|}
\hline Alumnado & Profesorado \\
\hline $\begin{array}{l}\text { - } \quad \text { Se adapta fácilmente por lo que ofrece } \\
\text { alternativas a todo el alumnado. } \\
\text { - } \quad \text { Mejora la tutela del alumnado durante su } \\
\text { aprendizaje ya que ofrece un seguimiento } \\
\text { individualizado. } \\
\text { - } \quad \text { Favorece el aprendizaje activo, significativo } \\
\text { y funcional. } \\
\text { - Evalúa todos los aspectos posibles. } \\
\text { - Resulta motivador, aumentando la } \\
\text { participación del alumnado en su proceso de } \\
\text { aprendizaje. } \\
\text { Está centrado en el proceso y da importancia } \\
\text { del trabajo diario. } \\
\text { Permite la retroalimentación continua y } \\
\text { formativa posibilitando la corrección de } \\
\text { errores, lo que revierte positivamente en la } \\
\text { calidad de los trabajos exigidos. } \\
\text { - Facilita la adquisición de competencias } \\
\text { profesionales y transversales. } \\
\text { Facilita que el alumnado aprenda más y que } \\
\text { obtenga un mayor rendimiento académico. }\end{array}$ & $\begin{array}{l}\text { - Permite que el profesorado conozca más y } \\
\text { mejora a sus estudiantes, hecho que } \\
\text { contribuye a que se ofrezca un } \\
\text { acompañamiento personal en su aprendizaje. } \\
\text { La metaevaluación que se produce cataliza } \\
\text { una serie de ajustes, regulaciones y } \\
\text { corrección de errores de la práctica docente } \\
\text { en general así como del proceso de } \\
\text { evaluación en particular. } \\
\text { - Requiere una planificación minuciosa, lo que } \\
\text { supone una mayor organización y coherencia } \\
\text { curricular, como consecuencia, posibilita } \\
\text { ofrecer una educación de más calidad. }\end{array}$ \\
\hline
\end{tabular}

En este artículo se presenta el fruto de un trabajo colaborativo entre 5 docentes universitarios encargados de impartir la asignatura de 'Didáctica de la Educación Física (EF) de la Educación Primaria', una materia obligatoria que se imparte en el segundo curso del Grado en Maestro/a de Educación Primaria de la Universidad de Valencia. En esta dirección, el trabajo colaborativo entre el profesorado y más concretamente, la investigación colaborativa, suponen una excelente herramienta de mejora de la formación y desarrollo profesional pues permite "que se cree una dinámica que hace más rico el trabajo del grupo" (Fraile, 2005: 58). Para ello, se ha seguido un procedimiento de ciclos de planificaciónacción-observación-reflexión sobre la práctica docente (Stenhouse, 1987; Tinning, 1992).

Los objetivos que nos proponemos pasan por compartir la experiencia, a través de una detallada descripción, para facilitar de este modo la aplicación en otras materias y grados universitarios y, cómo no, propiciar la reflexión sobre nuestra propia práctica docente.

\section{Presentación de la experiencia}

\subsection{Contexto}

Esta experiencia se ha ubicado en una materia cuatrimestral obligatoria en la que el alumnado del Grado en Maestro/a de Primaria todavía no ha elegido ninguna especialidad (esto ocurre 
en tercer y cuarto curso). En el curso 2013-2014 la facultad ha habilitado 11 grupos de la asignatura, de los cuales 5 han participado de la experiencia. Los grupos han cursado la materia tanto en el primer como en el segundo cuatrimestre. El número de alumnado ha oscilado entre 40 y 50 estudiantes en función del grupo. Dicho estudiantado ha de asistir a una asignatura en la que se trata de dar a conocer los fundamentos básicos de la EF, conocer y valorar el currículum, diseñar y aplicar tareas y sesiones específicas o adquirir recursos para fomentar hábitos saludables a lo largo de la vida, entre otros objetivos.

\subsection{Planteamiento didáctico}

La asignatura consta de 6 créditos $\mathrm{ECTS}^{4}$, de los cuales 2,4 corresponden a sesiones presenciales y 3,6 a sesiones no presenciales. En la Tabla 2 podemos encontrar la estimación de la carga de trabajo del alumnado en función de la distribución horaria que establece la Guía Docente de la asignatura .

Tabla 2. Distribución de la dedicación en la asignatura 'Didáctica de la EF de la Educación Primaria' por parte del alumnado.

\begin{tabular}{lc}
\hline & $\mathrm{n}^{\mathrm{o}}$ de horas \\
\hline Asistencia a clases teórico-prácticas & 60 \\
Preparación de trabajos & 28 \\
Preparación y presentación clases prácticas y exposiciones & 35 \\
Asistencia a tutorías & 15 \\
Preparación de exámenes & 12 \\
\hline Total volumen de trabajo & 150 \\
\hline
\end{tabular}

Dentro de la categoría 'Preparación de trabajos', el profesorado implicado acordó como actividad principal un proyecto de aprendizaje tutorado (PAT) consistente en la elaboración por grupos de una unidad didáctica (UD) relacionada con algunos de los contenidos propios de la EF y el desarrollo práctico de una de las sesiones diseñadas en el Aula de EF de la Facultad de Magisterio (pabellón deportivo).

\footnotetext{
${ }^{4}$ Los créditos ECTS equivalen normalmente a 25 horas de Trabajo del estudiante, por lo que no solo computan las horas lectivas.
} 


\subsection{Los itinerarios de evaluación}

Los itinerarios en evaluación se vienen usando con cierta recurrencia dentro de lo que entendemos por evaluación formativa o evaluación para el aprendizaje. El establecimiento de dichos itinerarios responde, por un lado, a la nueva concepción educativa que focaliza la atención sobre los procesos de aprendizaje en base a la participación del alumnado en dichos procesos y, por otro, a la necesidad de adaptar la propia evaluación de las materias a las distintas realidades que encontramos en las aulas. Obviamente, la implementación concreta de esta iniciativa, más allá de las generalidades, deberá mostrar elementos constitutivos específicos, adaptados a las concepciones y necesidades de cada realidad, del profesorado y del alumnado. En nuestro caso, el profesorado participante de esta iniciativa llegamos a varios acuerdos mínimos, dejando el resto de detalles sometidos a la voluntad y exigencias de docentes y discentes en cada caso.

De este modo, la propuesta de evaluación genérica quedó tal y como recoge la Tabla 3. Se trata de un acuerdo de mínimos que cada docente debía concretar y adaptar al grupo al que impartía clase (por ejemplo determinando técnicas, medios e intrumentos de evaluación). Cabe aclarar que la naturaleza de las preguntas de los exámenes variará según la vía escogida. 
Tabla 3. Acuerdos tomados para cada uno de los itinerarios ofertados al alumnado que concreta cada docente en su grupo.

\begin{tabular}{|c|c|c|c|}
\hline 赵 & Dirigido a & $\begin{array}{l}\text { \% sobre } \\
\text { la nota } \\
\text { final }\end{array}$ & Actividades y tareas que comprende \\
\hline \multirow{3}{*}{ 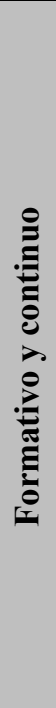 } & \multirow{3}{*}{$\begin{array}{l}\text { Alumnado que } \\
\text { asiste } \\
\text { regularmente a } \\
\text { clase }\end{array}$} & $40 \%$ & $\begin{array}{l}\text { Proyecto de aprendizaje tutorado: elaboración grupal de } \\
\text { una UD, exposición oral y desarrollo práctico de una } \\
\text { sesión. }\end{array}$ \\
\hline & & $40 \%$ & $\begin{array}{l}\text { Elaboración individual y grupal de distintos trabajos y } \\
\text { actividades: } \\
\text { - Participación en el blog de la asignatura. } \\
\text { - Elaboración de actividades en clase. } \\
\text { - Elaboración de actividades en casa. } \\
\text { - Exposiciones orales. } \\
\text { - Asistencia y participación en las clases prácticas. }\end{array}$ \\
\hline & & $20 \%$ & $\begin{array}{l}\text { Examen (el profesorado escoge una de las tres opciones): } \\
\text { a) Preguntas desarrolladas a lo largo del curso a través } \\
\text { del blog de la asignatura. } \\
\text { b) Preguntas y supuestos prácticos a contestar con } \\
\text { apuntes. } \\
\text { c) Preguntas a contestar sin apuntes. }\end{array}$ \\
\hline \multirow{2}{*}{ 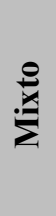 } & \multirow{2}{*}{$\begin{array}{l}\text { Alumnado que } \\
\text { no pueda } \\
\text { asistir al } 80 \% \\
\text { de las horas } \\
\text { presenciales }\end{array}$} & $40 \%$ & $\begin{array}{l}\text { Elaboración y defensa oral individual de una UD ante el } \\
\text { profesor/a. }\end{array}$ \\
\hline & & $60 \%$ & Examen. Preguntas de carácter teórico y práctico \\
\hline 焉 & $\begin{array}{l}\text { Alumnado que } \\
\text { no vaya a clase }\end{array}$ & $100 \%$ & Examen. Preguntas de carácter teórico y práctico \\
\hline
\end{tabular}

\section{Hallazgos más destacables}

En el itinerario de evaluación continua y formativa, por otra parte escogido por la mayoría del alumnado (88\%), el acuerdo se cerró en la enumeración de las actividades de aprendizaje a desarrollar por el alumnado y en los porcentajes de calificación, mientras que las tareas concretas que implicaba cada actividad se dejaron a libre elección en cada caso, aunque muchas de ellas han sido desarrolladas por varios profesores en sus respectivos grupos. Conviene destacar que, tal y como exige la evaluación formativa, se acordó que la elaboración de la UD fuera lo más tutorizada posible para poder facilitar al alumnado el feed- 
back necesario con el objetivo de que fuera mejorando su propuesta. Así, se habló igualmente de posibilitar dichas rectificaciones tanto en las exposiciones orales en las que se describían las unidades didácticas como en el desarrollo práctico de una de las sesiones diseñadas en las mismas. De igual manera, se decidió la introducción de prácticas de evaluación entre iguales (durante el proceso) y autoevaluación (al final del mismo) como ingredientes fundamentales de la evaluación formativa (Fraile \& Cornejo, 2012). Para ello el alumnado dispuso de una serie de intrumentos de evaluación como rúbricas y escalas de nivel. En el caso del examen, cada profesor escogió la opción que mejor se ajustara a las necesidades de su alumnado.

La propuesta mixta, por la que finalmente no se decantó ningún alumno o alumna, supone un paso intermedio entre la formativa y la final y trata de dar cierta oportunidad de participación en las actividades recurrentes de clase a las personas que, aun estando interesadas en la asistencia a la universidad, se vean impedidas a hacerlo de forma regular. Con esta opción, tienen la oportunidad de elaborar una UD que deberá ser expuesta ante el profesor/a.

Por último, el itinerario final, escogido por el 12\% del alumnado, está pensado para las personas que no quieren o no pueden asistir a clase y limitan su evaluación a un examen final de carácter teórico (donde se incluyen preguntas sobre los fundamentos didácticos de la EF) y práctico (relacionadas con las competencias de diseño de procesos y actividades de enseñanza-aprendizaje).

La decisión sobre en qué itinerario ubicarse corresponde a cada alumno y alumna, una vez se explica y se consensua el sistema de evaluación en los primeros días de clase, sin embargo, el incumplimiento de los requisitos establecidos para cada itinerario (asistencia mínima, presentación de las actividades de aprendizaje, etc.) puede hacer que finalmente se pase de un itinerario continuo y formativo o mixto a uno final. De igual manera se refleja la posibilidad de pasar de un itinerario continuo y formativo a uno mixto, en este último caso estudiante y docente deden valorar si es viable esta opción o pasar directamente a la evaluación final. 


\section{Conclusiones}

Tras la puesta en práctica de estas experiencias educativas basadas en la evaluación formativa y compartida, todos los docentes implicados coincidimos en destacar la bondad de este tipo de iniciativas tanto para la mejora de los procesos de aprendizaje del alumnado, y por ende de su educación, como de las formas concretas en que el profesorado diseña sus enseñanzas. La continua experimentación en el diseño de las asignaturas, junto a la reflexividad que le otorga a este proceso el trabajo en colaboración con compañeros y compañeras, son una excelente garantía de mejora profesional, en parte, como apuntan Azaustre y Monescillo (2013) para tomar conciencia de la importancia de la evaluación y poner en valor formas alternativas de implementarla. Ante esto y como líneas de futuro, nos planteamos ir puliendo la propuesta para reducir nuestra carga de trabajo, adaptar mejor las actividades a las competencias que se le asignan al grado en cuestión, otorgar mayor participación si cabe al alumnado y, por supuesto, difundir la iniciativa para, con ello, hacerla más interdisciplinaria. En este sentido, una de las áreas que se nos revela más interesante corresponde a las actividades de autoevaluación y evaluación compartida, más arriba nombradas, y que cada docente ha concretado de forma diferente en su grupo. Se trata, por tanto, de una línea a seguir para la generalización de una evaluación más democrática y educativa, en la que los itinerarios de evaluación suponen un paso significativo.

\section{Referencias bibliográficas}

Arribas, J.M. (2012). El rendimiento académico en función del sistema de evaluación empleado. Revista Electrónica de Investigación y Evaluación Educativa (RELIEVE), 18(1), 1-15. Consulta realizada 8 abr. 2014. http://www.uv.es/RELIEVE/v18n1/RELIEVEv18n1_3.pdf

Azaustre, C., \& Monescillo, M. (2013). La evaluación formativa en el contexto educativo. Orientaciones desde la práctica docente. Revista de Evaluación Educativa, 2(2), 1 23. Consulta realizada $11 \quad 2014$. http://revalue.mx/revista/index.php/revalue/article/view/58/150

Benito, A., \& Cruz, A. (2003). Nuevas claves para la docencia universitaria en el Espacio Europeo de Educación Superior. Madrid: Narcea. 
Biggs, J. (2005). Calidad del aprendizaje universitario. Madrid: Narcea.

Burcà, F., Pintor, P., Martínez, L., \& Peire, T. (2010). Sistemas y procedimientos de evaluación formativ en docencia universitaria: resultados de 34 casos aplicados durante el curso académico 2007-2008. Estudios sobre educación, 18, 255-276.

Fraile, A. (2005). La formación colaborativa en el profesorado de Educación Física. En A. Sicilia \& J.M. Fernández-Balboa (coords.), La otra cara de la enseñanza. La Educación Física desde una perspectiva crítica (pp. 49-75). Barcelona: Inde.

Fraile, A., \& Cornejo, P.V. (2012). La evaluación formativa en la enseñanza universitaria: Una experiencia de innovación educativa con estudiantes de Educación Física. Revista de Evaluación Educativa, 1(2), 22-43. Consulta realizada 13 marz. 2014. http://revalue.mx/revista/index.php/revalue/article/view/36/103

Gijón, J., \& Crisol, E. (2012). La internacionalización de la Educación Superior. El caso del Espacio Europeo de Educación Superior. Revista de Docencia Universitaria. REDU, 10(1), 389-414. Consulta realizada 10 abr. 2014. http://dialnet.unirioja.es/descarga/articulo/4020541.pdf

Hamodi, C. (coord.). (2016). Formar mediante la evaluación en la Universidad. Propuestas prácticas útiles para docentes. Valladolid: Universidad de Vaslladolid.

López-Partor, V.M. (2009). Evaluación Formativa y Compartida en Educación Superior. Propuestas, técnicas, instrumentos y experiencias. Madrid: Narcea.

López-Pastor, V.M. (2011). Aclaraciones terminológicas sobre evaluación y sus tipos. A modo de glosario e introducción. En V.M. López-Pastor (Coord.), La evaluación en educación física (pp. 21-33). Buenos Aires: Miño y Dávila.

López-Pastor, V.M., Castejón, J., Sicilia-Camacho, A., Navarro-Adelantado, V. \& Webb, G. (2011). The process of creating a cross-university network for formative and shared assessment in higher education in Spain and its potential Applications. Innovations in Education and Teaching International, 48(1), 79-90.

López-Pastor, V.M., Pintor, P., Muros, B., \& Webb, G. (2013). Formative assessment strategies and their effect on student performance and on student and tutor workload: 
the results of research projects undertaken in preparation for greater convergence of universities in Spain within the European Higher Education Area (EHEA). Journal of Further and Higher Education, 37(2), 163-180.

Manrique, J.C., Vallés, C, \& Gea, J.M. (2012). Resultados generales de la puesta en práctica de 29 casos sobre el desarrollo de sistemas de evaluación formativa en docencia universitaria. Psychology, Society \& Education, 4(1), 87-102.

Vallés, C., Ureña, N., \& Ruíz, E. (2011). La Evaluación Formativa en Docencia Universitaria. Resultados globales de 41 estudios de caso. REDU. Revista de Docencia Universitaria, 9(1), 136-157.

Stenhouse, L. (1978). La investigación como base de la enseñanza. Madrid: Morata.

Tinning, R. (1992). Educación fisica: La escuela y sus professores. Valencia: Servei de publicacions Universitat de València.

Zaragoza, J., Luis-Pascual, J.C., \& Marnrique, J.C. (2009). Experiencias de innovación en docencia universitaria: resultados de la aplicación de sistemas de evaluación formativa. REDU. Revista de Docencia Universitaria, 4, 1-33. 